\title{
Sodium Alginate Microspheres
}

National Cancer Institute

\section{Source}

National Cancer Institute. Sodium Alginate Microspheres. NCI Thesaurus. Code C97515.

An embolic agent containing microporous hydrospheres of sodium alginate with potential arterial occlusive activity. In transarterial chemoembolization (TACE), the sodium alginate microspheres (KMG) are administered into blood vessels that feed the tumor, occluding tumor blood vessels and inducing ischemic tumor necrosis. In addition, these microspheres may be used to encapsulate various therapeutic agents. 